\title{
Las enseñanzas artísticas superiores y el Espacio Europeo de Educación Superior en España. Una lectura crítica ${ }^{1}$
}

\section{Higher artistic education and the European Higher Education Area in Spain. A critical appraisal}

\author{
Manuel Francisco VIEITES ${ }^{2}$ \\ Escuela Superior de Arte Dramático de Galicia
}

Recibido: Septiembre 2014

Evaluado: Noviembre 2014

Aceptado: Noviembre 2014

\section{Resumen}

El Real Decreto 1614/2009, de 26 de octubre, que establecía la ordenación de las enseñanzas artísticas superiores reguladas por la Ley Orgánica 2/2006, de 3 de mayo, de Educación, señalaba que dichas enseñanzas debieran converger en el Espacio Europeo de Educación Superior. Pasados cuatro años desde la implantación de los nuevos planes de estudio y egresada la primera promoción formada al amparo de la Declaración de Bolonia, analizamos ahora en qué medida se ha producido ese proceso de convergencia atendiendo a parámetros utilizados en otras instituciones de formación superior como las universidades, a partir de la legislación aplicable y de las medidas que se han adoptado tanto a nivel ministerial como en las comunidades autónomas. Finalmente ofrecemos una propuesta que permita hacer de esa integración un punto de inflexión en el fomento y puesta en valor de unas enseñanzas fundamentales en el desarrollo de nuestro sistema cultural.

Palabras clave: enseñanzas artísticas, espacio europeo de educación superior, legislación educativa, convergencia europea.

\footnotetext{
${ }^{1}$ Este texto se vincula al Proyecto de Investigación "De los tiempos educativos a los tiempos sociales: la construcción cotidiana de la condición juvenil en una sociedad de redes. Problemáticas y alternativas pedagógico-sociales", del que es IP José Antonio Caride Gómez, subvencionado mediante convocatoria pública por la Secretaría de Estado de Investigación, Desarrollo e Innovación del Ministerio de Economía y Competitividad, Gobierno de España, en el marco del Plan Nacional de I+D+i (convocatoria 2012), con el código EDU2012-39080-CO7-01, con financiamiento de la Unión Europea a través de los fondos FEDER.

${ }^{2}$ El autor es profesor y director de la ESAD de Galicia, y profesor asociado de la Universidad de Vigo, en el Departamento de Análisis e Intervención Psicosocioeducativa, Facultad de Ciencias de la Educación, Orense.
} 


\begin{abstract}
The Royal Decree Act 1614/2009 of 26 October, which ordained the higher artistic education regulated by the Organic Act 2/2006, of 3 May 2006, on Education, established that their degrees should converge on the European Higher Education Area. After four years of implementation of the new curriculum and after the graduation of the first students under the Bologna Accords, we explore to what extent this process of convergence has taken place according to parameters used in other higher education institutions such as universities, considering the applicable law and the measures that have been adopted both at ministerial level and in the autonomous regions. Finally, we offer a proposal that allows this integration to become a turning point in the promotion and enhancement of a field of education fundamental in the development of our cultural system.
\end{abstract}

Keywords: Artistic Education, European Higher Education Area, Educative Legislation, European Convergence.

La puesta en marcha del Espacio Europeo de Educación Superior (EEES) generó un rico e intenso debate en la comunidad académica universitaria, que se refleja en el elevado número de libros, artículos en revistas especializadas, o tesis doctorales, que se pueden consultar en bases de datos como DIALNET o TESEO. Ahí encontraremos desde trabajos que analizan el proceso de transformación de una titulación, hasta otros que se ocupan de cuestiones metodológicas pertinentes al nuevo marco. No abundan, sin embargo, los trabajos que se ocupen de la integración de las enseñanzas artísticas superiores en ese mismo espacio, salvo excepciones (Pérez García y Sicilia, 2011; Pastor Gordero, 2012; Calvet, 2013; Luna 2014; Vieites, 2015b).

De hecho, en 2003, el Ministerio de Educación, Cultura y Deporte (MECD) publicaba el documento marco La integración del sistema universitario español en el Espacio Europeo de Educación Superior, en el que no se realizaba ninguna referencia a las enseñanzas artísticas superiores, las que la Ley General de Educación de $1970^{3}$ quiso situar en la universidad y que finalmente quedarían fuera, por razones no explicadas. En efecto, la llamada Ley Villar Palasí, en su disposición adicional segunda, apartado cuatro, establecía que "las Escuelas Superiores de Bellas Artes, los Conservatorios de Música y las Escuelas de Arte Dramático se incorporarán a la educación universitaria en sus tres ciclos, en la forma y con los requisitos que reglamentariamente se establezcan" (p. 12543). Finalmente, sólo las Escuelas de Bellas Artes iniciarían el proceso con lo que las enseñanzas artísticas de arte dramático, danza, diseño, música, o conservación y restauración de bienes culturales, se mantendrían al margen de la universidad; con todo, existen en la universidad española (pública, y especialmente en la privada) grados oficiales o títulos propios en esas enseñanzas y con denominaciones idénticas o similares.

En aquel entonces se dejaron oír voces (Castro, 1969) que reclamaban una incorporación a la universidad que se tornó empresa imposible por dos razones fundamentales; de un lado, una visión sumamente negativa que los centros superiores

\footnotetext{
${ }^{3}$ Boletín Oficial del Estado de 6 de agosto de 1970. Disponible en: https://www.boe.es/boe/dias/1970/08/06/pdfs/A12525-12546.pdf 
fueron construyendo de la universidad, y, del otro, el poco interés que mostraron las administraciones educativas en el cumplimiento o desarrollo de las normas que ellas mismas habían dictado. Es necesario decir que, en lo que atañe a las enseñanzas artísticas superiores, el de la LGE de 1970 sería el primero de una larga serie de incumplimientos que derivan en la situación actual (Carrera, 2014).

Las enseñanzas artísticas superiores siguen desde 1970 un proceso de desarrollo que culmina en 1990 con la Ley de Ordenación General del Sistema Educativo (LOGSE) que establecía la equivalencia de sus titulaciones a licenciaturas y diplomaturas universitarias. Además, para las enseñanzas superiores de arte dramático, danza y música, la LOGSE contemplaba la posibilidad de organizar estudios de tercer ciclo mediante convenio con las universidades. La Ley Orgánica de Educación, de 2006, las equiparaba al título universitario de grado, manteniendo abierta la posibilidad de la organización de estudios de doctorado "propios de las enseñanzas artísticas reguladas" en la propia Ley, mientras en su artículo 58.6 establecía que "los centros superiores de enseñanzas artísticas fomentarán programas de investigación en el ámbito de las disciplinas que les sean propias" (p. 17177) ${ }^{4}$.

En 2009 se publicaba el Real Decreto $1614 / 2009^{5}$ que regulaba como títulos de "grado" en artísticas superiores los que la LOE definía como "títulos superiores", equivalentes al grado universitario. Además, explicitaba la necesaria integración de los estudios en el EEES y todo un conjunto de intenciones que se trasladarían a los Reales Decretos 630/2010, 631/2010, 632/2010, 633/2010, 634/2010 y 635/2010 (citamos por 630/2010) ${ }^{6}$; éstos regulaban aspectos básicos del currículo de los estudios superiores de arte dramático, música, danza, diseño, vidrio y cerámica, y conservación y restauración de bienes culturales, y otras cuestiones vinculadas con el dicho EEES.

Pero en enero de 2012 se hacía pública una sentencia del Tribunal Supremo ${ }^{7}$ en la que se aceptaba en parte el recurso presentado por la Universidad de Granada en la que se cuestionaba el hecho de que centros que no eran universitarios pudiesen ofrecer titulaciones de grado y posgrado (máster) (Marzal, 2012). La sentencia eliminaba la denominación de grado que contemplaba el RD 1614/2009, y poco después la Ley Orgánica 8/2013 para la Mejora de la Calidad Educativa (LOMCE) recuperaba la denominación de "título superior" para lo que había sido fugazmente grado, con lo que las artísticas superiores volvían al punto de partida, a la equivalencia establecida en 1990 por la LOGSE, si bien con el añadido de que tanto la LOE como la LOMCE ubican tales enseñanzas en el Espacio Europeo de Educación Superior (Lemes, 2012).

Pese a todo, la cuestión del EEES y de su transcendencia para las artísticas superiores no fue objeto de estudios substantivos. Así, el MECD publicaba un monográfico titulado Las Enseñanzas Artísticas Superiores en el Espacio Europeo de Educación Superior (López, Vico et al., 2010) que no aportaba mayores novedades

\footnotetext{
${ }^{4}$ LOE, BOE del 4 de mayo de 2006.

${ }^{5}$ BOE de 27 de octubre de 2009.

${ }^{6} \mathrm{BOE}$ de 5 de junio de 2010.

${ }^{7}$ Fueron varias sentencias, dado que varias universidades impugnaron el RD 1614/2009. La más completa aparece recogida en el BOE de 21 de diciembre de 2012, en su página 86883.
} 
que las establecidas en la normativa que en aquellos momentos entraba en vigor. Un año después el Consejo Superior de Enseñanzas Artísticas (CSEA) emitía un Informe anual sobre el estado y situación de las Enseñanzas Artísticas, en el que, como novedad, destacaba la necesidad de dotar a los centros de un nuevo marco de organización y funcionamiento, de acuerdo con el nuevo perfil que el citado RD 1614/2009 les confería, normativa inexistente a día de hoy. Como norma (Zaldívar, 2005; Bas Palmero, 2014) la cuestión del EEES se entendió de forma puramente nominal, como si la simple invocación del sintagma o de su acrónimo supusiese un avance significativo en el proceso de desarrollo de las enseñanzas, olvidando todo aquello que el sintagma implica y demanda (ACESEA, 2012).

Por todo ello, en este artículo queremos analizar qué sentido se le ha dado al EEES en el ámbito de las enseñanzas artísticas superiores (EEAASS) en la legislación específica aplicable (ACESEA, 2010), y cuáles han sido las iniciativas de las autoridades académicas para la integración de las enseñanzas en ese nuevo marco, para finalmente proponer líneas de trabajo que faciliten y garanticen esa convergencia.

\section{El Espacio Europeo de Educación Superior en la normativa de las EEAASS}

La normativa específica aplicable en relación a la confluencia de las artísticas superiores en el EEES se encuentra contenida básicamente en el RD 1614/2009 y en los Reales Decretos antes citados $(63 * / 2010)$. Como veremos, en la misma se proponen "cambios legales o administrativos", "cambios institucionales", y "cambios en la docencia", similares a los que afectaron a las enseñanzas universitarias (Zabalza, 2008a, p. 201). Se concretan en:

- Crédito europeo, pues "el haber académico que representa el cumplimiento de los objetivos previstos en los planes de estudios conducentes a la obtención de títulos oficiales de enseñanzas artísticas superiores se medirá en créditos europeos ECTS" (RD 1614/2009, p. 89745).

- Aprendizaje autónomo, dado que en la asignación de horas de trabajo por asignatura se consideran tanto las lectivas como las horas de estudio, y "las dedicadas a la realización de seminarios, trabajos, prácticas y proyectos" (RD 1614/2009, p. 89745).

- Competencias, muy vinculadas con el saber hacer y que confieren a las asignaturas una dimensión esencialmente práctica, que exige la "aplicación de una nueva metodología de aprendizaje, y en la adecuación de los procedimientos de evaluación" (RD 630/2010, p. 48467).

- Un nuevo modelo de docencia, pues el RD 1614/2009 habla de "un cambio estructural basado en la flexibilidad en la organización de la enseñanza y la renovación de las metodologías docentes, cuyo objetivo se centra en el proceso de aprendizaje del estudiante" (p. 89743). 
- Formación del profesorado, al decir que "las Administraciones educativas propiciarán planes de formación del profesorado, relativos al conocimiento de los principios básicos, la estructura, la organización, las nuevas metodologías y los sistemas de evaluación e investigación correspondientes al Espacio Europeo de Educación Superior". Además se señala que "las Administraciones educativas, a propuesta de los centros, fomentarán planes de formación del profesorado para la actualización y profundización de las disciplinas propias... en sus diferentes ámbitos" (RD 630/2010, pp. 48471 y 48472).

- Estructura de las enseñanzas, que van del grado al posgrado, con un máster en enseñanzas artísticas, "de carácter especializado o multidisciplinar", orientado "a la especialización académica o profesional, o bien a promover la iniciación en tareas investigadoras". Se propone un doctorado propio, en convenio con las universidades, que deberá "incluir los criterios de admisión y las condiciones para la realización y elaboración de la tesis doctoral y su adecuación a las particularidades de las enseñanzas artísticas superiores entre las que se podrá considerar la interpretación y la creación" (RD 1614/2009, p. 89747).

- Calidad y evaluación, para "mejorar la actividad docente, investigadora y de gestión de los centros, así como fomentar la excelencia y movilidad de estudiantes y profesorado" (RD 1614/2009, p. 89751).

- Autonomía de los centros, en los "ámbitos organizativo, pedagógico y de gestión" (RD 1614/2009, p. 89743). En la misma dirección, se dice que "las Administraciones educativas favorecerán la autonomía pedagógica, de organización y de gestión de la que disponen estos centros para el ejercicio de sus actividades docentes, investigadoras, interpretativas y de difusión del conocimiento, a fin de garantizar el cumplimiento de sus funciones como centros educativos superiores del Espacio Europeo de Educación Superior" (RD 630/2010, p. 48468).

- Recursos, en tanto se dice que las administraciones educativas "dotarán a los centros de enseñanzas artísticas superiores de los recursos necesarios para facilitar su funcionamiento, desarrollar sus objetivos en las áreas de la docencia, la investigación y la creación artística" (RD 1614/2009, p. 89743).

- Investigación, pues se establece que "los centros de enseñanzas artísticas superiores... fomentarán programas de investigación científica y técnica propios" de su campo disciplinar, "para contribuir a la generación y difusión del conocimiento y a la innovación en dicho ámbito". También se afirma que "las Administraciones educativas establecerán los mecanismos adecuados para que estos centros puedan realizar o dar soporte a la investigación científica y técnica, que les permita integrarse en el Sistema Español de Ciencia y Tecnología" (RD 630/2010, p. 48468).

- Investigación y creación, por cuanto se afirma que "la consolidación de líneas de investigación y creación, la formación de creadores e investigadores son aspectos imprescindibles a fomentar y considerar por parte de las instituciones 
públicas y privadas". Además, se señala que "la colaboración entre centros enmarcados en el contexto de la educación superior es una necesidad de crecimiento y desarrollo conjunto en el ámbito de la docencia y de la investigación" (RD 630/2010, p. 48467).

- Movilidad, al establecer que "las Administraciones educativas facilitarán el intercambio y la movilidad de los estudiantes, titulados y profesorado de las enseñanzas... en el Espacio Europeo de Educación Superior, dentro de los programas europeos existentes conforme su propia normativa" (RD 630/2010, p. 48471).

De la lectura de la legislación aplicable es fácil deducir que la norma explicita funciones y responsabilidades para los centros, el profesorado, y el alumnado, similares a lo que el EEES demandaba de las universidades (Rué, 2004; Zabalza, 2008b). Todo ello implica un cambio substantivo en la concepción misma de los centros y en su estructura organizativa, para potenciar la adaptación a ese nuevo marco, en tanto éstos, con la actual legislación, tienen la consideración de institutos de educación secundaria, por cuya normativa se rigen. Cabría agrupar esas funciones y responsabilidades en cuatro grandes apartados:

- Nuevo modelo de currículo, con cambios substantivos en la concepción de los procesos educativos: docencia, aprendizaje, creación, investigación, e igualmente en la orientación de los mismos, vinculados con la praxis profesional y la transición a la vida activa.

- Nuevo modelo de centro, con mayor autonomía en la gestión y con los recursos necesarios para el desarrollo de nuevas funciones, o para el rendimiento de cuentas a través los sistemas internos de garantía de la calidad.

- Nuevo modelo de alumnado, en tanto el peso del trabajo autónomo configura un modelo diferente de organizar el aprendizaje.

- Nuevo modelo de profesorado, que supone promover la carrera investigadora y creativa de los docentes, que ha de estar necesariamente vinculada con la praxis artística, pero igualmente con una investigación centrada en el desarrollo de cada una de las disciplinas que integran el currículo en sus dimensiones histórica, teórica, metodológica, práctica, e incluso tecnológica (Vieites, 2015a).

La cuestión estaría entonces en determinar en qué medida las administraciones educativas han desarrollado líneas de actuación para enfrentar con éxito esas profundas transformaciones. De partida, diremos que una búsqueda sistemática por los boletines oficiales de las Comunidades Autónomas y de las páginas web de Departamentos o Consejerías de educación de las mismas, ofrece muy pocos resultados, por lo que podemos afirmar que apenas se ha desarrollado una normativa específica. 


\section{El Espacio Europeo de Educación Superior en la práctica}

Pese a los buenos augurios (Font, 2010), la integración de las enseñanzas artísticas superiores en el EEES se limita al hecho de que el alumnado recibe una titulación equivalente a todos los efectos al grado universitario, y se favorece la movilidad mediante el programa ERASMUS. En todo lo demás, la situación deja mucho que desear y algunas razones hay que buscarlas en el hecho de que estas enseñanzas se impartan en centros que carecen de esa dimensión superior, y por un profesorado que pertenece a cuerpos del mismo nivel y complemento de destino que el profesorado de secundaria, al que, en ningún caso, se le reconoce la carrera investigadora o creadora, y que debe cumplir una jornada laboral que en nada la facilita.

\section{Estructura de los estudios}

Tras la sentencia del Tribunal Supremo de enero de 2012, la LOMCE establece que quienes finalicen los estudios artísticos superiores obtendrán un título superior equivalente a todos los efectos al título universitario de grado. Otro tanto señala el Real Decreto 96/2014, de 14 de febrero, que modifica el Real Decreto 1027/2011, de 15 de julio, por el que se establece el Marco Español de Cualificaciones para la Educación Superior, y que sitúa en el nivel 2 el título de graduado y el título superior en enseñanzas artísticas. Con todo, esa equivalencia puede resultar engañosa por cuanto una norma española no es de obligado cumplimiento en Europa. Además, puede ser interpretada de forma variada en España, pues hay egresados en especialidades de estudios con título superior y título de grado, como ocurre en conservación y restauración de bienes culturales o diseño. Esa duplicidad de titulaciones equivalentes podría afectar de forma negativa a los titulados superiores en bolsas de trabajo, becas, o estudios de posgrado, al no ser en ningún caso el título superior un título universitario, lo que sitúa al primero en situación de desventaja.

Otro tanto ocurre con el Suplemento Europeo al Título que se habrá de regular para las artísticas superiores, y que encuentra el problema de su traducción, para la que existen dos opciones: o bien Bachelor (BA), o bien Higher Degree, Higher Diploma, Advanced Degree, o Advanced Diploma. La primera no se podrá utilizar pues es la propia del "grado", y cualquiera de las otras cuatro puede generar confusión porque refieren títulos de formación profesional superior o títulos universitarios en desuso en todo el continente y que equivaldrían a una diplomatura.

Similares problemas plantean las enseñanzas de posgrado, pues si bien el RD 1614/2009 establece que los centros podrán ofrecer el "máster en enseñanzas artísticas”, equivalente al máster universitario, en la práctica aquél tiene una orientación profesional, y en ningún caso vinculada con los estudios de posgrado o con un título, el de doctor o doctora, que otorga la universidad. Es de señalar que el Real Decreto 99/2011, de 28 de enero, por el que se regulan las enseñanzas oficiales de doctorado, establece en su artículo 6 que para cursar dichos estudios los aspirantes habrán de estar en posesión del título de máster universitario u otros equivalentes, y 
entre estos últimos en ningún caso aparece el citado "máster artístico" (p. 13914), cuestión que no modifica el Real Decreto 534/2013, de 12 de julio".

Del mismo modo, los estudios de doctorado en las especialidades propias de las enseñanzas artísticas tienen a día de hoy difícil trámite, pues entre las áreas de conocimiento contempladas por el Ministerio de Educación, Cultura y Deporte ni aparecen las de arte dramático, conservación y restauración, danza, o diseño, ni existen departamentos o facultades con tal denominación. Se puede ofrecer un programa de doctorado en "Artes de la Escena", pero el título de doctor o doctora lo será en la facultad en que se haya defendido la tesis. En ese sentido, es de señalar que sólo en universidades privadas, en las que empiezan a florecer los grados en enseñanzas artísticas, se podrán obtener doctorados en danza, arte dramático o música.

Todo esto nos lleva a considerar en qué medida el alumnado de los centros superiores de enseñanzas artísticas disfruta derechos similares a los de sus homólogos universitarios, en cuestiones importantes como estructura de los estudios, titulaciones de posgrado, becas y otras ayudas, residencias, instalaciones, o recursos disponibles..., y la realidad demuestra que son sensiblemente inferiores. Así, muchos centros carecen de bibliotecas de investigación, de red wifi, de servicios de teledocencia, o de espacios para el trabajo autónomo, tan importantes en la enseñanza superior.

\section{¿Un nuevo modelo de profesorado?}

Atendiendo a la norma y a las declaraciones de principios estamos, en efecto, ante un nuevo marco. El crédito europeo o ECTS supone un nuevo modelo de docencia y de aprendizaje que implica trabajo autónomo del alumno y que el profesor pase a ser un mediador entre estudiantes y aprendizajes, para que el alumnado construya el conocimiento en el saber y en el saber hacer. Es importante señalar la importancia de esta unidad de medida en tanto el concepto de "actividad lectiva" se amplía a las "actividades académicas dirigidas" y a otras formas de desarrollo de las tareas de docencia y aprendizaje (Menéndez Varela, 2009); el docente asume además funciones que hasta la fecha le eran ajenas, desde la tutoría académica (Gairín, Feixas, Guillamón y Quinquer, 2004) hasta la formación de investigadores (Morales, Rincón y Romero, 2005), un ámbito especialmente estratégico en enseñanzas artísticas, por la escasa tradición presente en los centros y en los propios estudios.

Las tutorías docentes aparecen como ese espacio de mediación que complementa el trabajo en el aula y en el que profesorado y alumnado realizan otras actividades (Álvarez Pérez y González Afonso, 2008). Atendiendo a la normativa vigente, el concepto de tutoría docente es ajeno a la organización académica de los centros de EEAASS, y el profesorado se limita a impartir sus 16, 18 o 20 horas lectivas de clase a la semana, que completa hasta las 23 de permanencia con tutorías de grupo, guardias de centro, gestión de biblioteca u otras actividades, siguiendo la pauta del profesorado

\footnotetext{
${ }^{8} B O E$ del 10 de febrero de 2011.

${ }^{9}$ BOE del 13 de julio de 2013. 
de secundaria, salvo excepciones ${ }^{10}$. Además, los centros carecen de las infraestructuras necesarias para que esa labor fundamental se pueda desarrollar con los criterios de eficacia, privacidad y disponibilidad de recursos con que cuentan las universidades.

La tutoría docente se justifica en función de la aplicación de la unidad de medida del haber académico denominada ECTS, que contempla actividades académicas dirigidas así como el seguimiento de los procesos de enseñanza y aprendizaje o el trabajo autónomo del alumnado (Álvarez González, 2008). Todo ello tiene que ver con la jornada laboral del profesorado y con la adecuación de ésta a ese nuevo perfil del profesor como docente, investigador y creador. En esa dirección, cabría partir del análisis que Arenas, Herrera y Díaz realizaban para la distribución posible de las 37,5 horas de jornada laboral del funcionario docente, en el que se computaban las dedicadas a investigación, docencia presencial, tutoría y otras actividades (2012, p. 56), y que en nuestro caso debieran tener, respectivamente, los valores de 10, 12, 6 y 9,50. Pues hay que señalar que la actual jornada laboral impide la movilidad y las estancias en centros de investigación o de creación, o que las normas para optar a licencias y permisos son sumamente restrictivas, por lo que asistir a un congreso, impartir un seminario o recibir un curso de formación se torna una tarea épica.

En la adecuación del profesorado al nuevo marco se ha dejado sentir con fuerza la inacción de las administraciones educativas. En el Informe anual sobre el estado y situación de las Enseñanzas Artísticas, se decía:

Las innovaciones docentes impuestas por el Espacio Europeo de Educación Superior exigen una formación muy especializada de modo que tanto la formación inicial como la permanente del profesorado garantice conocimientos adecuados, didácticas específicas y posibilite aplicar desde el aula las nuevas tecnologías para la elaboración y difusión de conocimientos, de modo que con el uso de estas herramientas, el docente pueda promover el aprendizaje activo -y no la mera transmisión de conocimiento- conforme a las nuevas metodologías del Espacio Europeo de Educación Superior (CSEA, 2011, p. 52).

Incluso se proponía que esa formación se organizase "a través de la suscripción de convenios con otras instituciones" (p. 52), que en buena lógica se suponen universitarias y que sí desarrollaron muy diferentes actividades al objeto de facilitar el tránsito al EEES.

Todo ello nos sitúa ante una problemática que puede ser analizada al menos en dos direcciones. Cabe preguntar si es razonable que profesorado con el mismo nivel y complemento de destino que el profesorado de secundaria imparta docencia en titulaciones equivalentes a un grado universitario. Y también cabe analizar en qué

\footnotetext{
${ }^{10}$ Así en la ORDEN de 8 de agosto de 2011 por la que se desarrolla el Decreto 61/2011, de 24 de marzo, por el que se establece el Reglamento orgánico de las escuelas de arte y superiores de diseño de la Comunidad Autónoma de Galicia, publicada en el Diario Oficial de Galicia con fecha del 18 de agosto de 2011, se reconocen como docencia directa hasta un máximo de tres horas semanales de tutoría docente.
} 
medida ese profesorado puede, como demanda la normativa actual, desarrollar una carrera docente, investigadora y creadora, para la que probablemente esté plenamente capacitado, aunque carezca de las herramientas administrativas para demostrarlo, en tanto en su perfil profesional ni se contempla la investigación ni la creación, y por tanto tampoco su acreditación.

\section{La paradoja de los centros: enseñanzas superiores en institutos de secundaria}

El Informe anual sobre el estado y situación de las Enseñanzas Artísticas señalaba que en el ámbito organizativo bueno sería aprovechar "la experiencia acumulada en el funcionamiento de los Institutos Superiores de Enseñanzas Artísticas u organismos similares" por lo que se aconsejaba, "la generalización de estos organismos que con carácter autónomo facilite la gestión para fomentar la empleabilidad, la movilidad, la formación de grado y de postgrado y los instrumentos multidimensionales de transparencia y financiación" (CSEA, 2011, p. 54). Infelizmente tales Institutos, presentes en la Comunidad Valenciana o en Andalucía, en ningún caso pueden desarrollar normativas o funciones equiparables a la de otras instituciones superiores, pues no tienen rango de universidades sino de direcciones generales. En el caso del Instituto Andaluz de Enseñanzas Artísticas Superiores, éste se concibe como una "agencia administrativa"11, no como una institución educativa. Toda una declaración programática.

Una muestra de esa falta de adecuación al EEES, la tenemos en la ordenación de la jornada laboral del profesorado del Instituto Superior de Enseñanzas Artísticas de la Comunidad Valenciana (ISEACV), al contemplar un total de 20 horas lectivas semanales, de las que hasta un total de 3 se podrán dedicar a proyectos de investigación presentados por los departamentos y aprobados por la dirección del Instituto (ISEACV, 2013), aunque esa investigación no se vincule en ningún caso con la que se promueve al amparo del Sistema Español de Ciencia y Tecnología, tal y como demandan los Reales Decretos 630 a 635/2010. De igual modo, el profesorado de centros superiores de enseñanzas artísticas que cuenta con titulación de doctor y con trayectoria investigadora no puede verificarla ante instancias competentes, lo que es una de las causas, aunque no la única, de que no pueda dirigir tesis doctorales ni equipos de investigación financiados al amparo de convocatorias oficiales de I+D+i.

En otros casos se han dado pasos que, en la práctica no han supuesto más que cambios en la administración y supervisión de centros. Eso ocurre en la Comunidad de Madrid, en la que las enseñanzas artísticas superiores pasan a depender de la Dirección General de Universidades e Investigación sin que ello suponga desarrollos normativos

${ }^{11}$ Ley 17/2007, de 10 de diciembre, de Educación de Andalucía, publicada en el BOJA, no 252, de 26 de diciembre, p. 20. 
que sirvan para afrontar los desafíos antes señalados, con lo que la medida no supone más que un simple cambio de ubicación de una Subdirección General ${ }^{12}$.

Como señalaba López, la enseñanza universitaria no es la única enseñanza superior, pero por ser superior requiere un marco de organización diferenciado, en función de su naturaleza:

Los profesionales de las distintas enseñanzas artísticas y quienes han estudiado sobre su posible ordenación, dentro y fuera de las Administraciones educativas, reparten sus opiniones entre la opción de integrar estas enseñanzas dentro de las universidades o la opción de configurar un espacio propio de educación superior. Ambas opciones tienen ventajas y tienen inconvenientes. La inclusión de estas enseñanzas en la LOE, en la medida en que ésta no regula la enseñanza universitaria, comporta la decisión de abordar la creación de un espacio específico de educación superior para las enseñanzas artísticas (2010, pp. 15-16).

El problema es que ese espacio específico no se ha creado, pues si bien se han generado estructuras institucionales, éstas carecen de capacidad para promover un ordenamiento que vaya más allá del marco de la educación secundaria o de la formación profesional.

Existen otras necesidades y demandas, que afectan a las infraestructuras y a los recursos, y de nuevo habría que hacer referencia a otros incumplimientos. Así el RD 389/1992, de 15 de abril, que establecía los requisitos mínimos de los centros superiores de enseñanzas artísticas, señalaba en su artículo 18.3 que los conservatorios superiores de música debieran contar con:

Biblioteca, videoteca y fonoteca. Deberá permitir en el conjunto de sus secciones, la utilización simultánea de al menos, un 5 por 100 del número total de alumnos previstos. Dispondrá de sala de lectura, sala de audición y vídeo, archivo y sistema de préstamo. Quedará garantizado el número de volúmenes y grabaciones necesario para el correcto desarrollo de las enseñanzas que se impartan y su uso en soporte no convencional, así como de las principales revistas científicas relacionadas con el ámbito de dichas enseñanzas ( $\mathrm{p}$. $14154)^{13}$.

Lo mismo se decía, por ejemplo, para el caso de arte dramático, conservación y restauración, danza o diseño, sin que esas instalaciones y dotaciones, salvo en centros con una larga trayectoria como la RESAD de Madrid, el Institut del Teatre de Barcelona o el Real Conservatorio Superior de Música de Madrid, se llegasen a concretar en el período de vigencia del Real Decreto, entre 1992 y 2010. En este último

12 Decreto 126/2012, de 25 de octubre, del Consejo de Gobierno, por el que se establece la estructura orgánica de la Consejería de Educación, Juventud y Deporte, publicado en el BOCM, n ${ }^{\circ} 256$, de 26 de octubre.

${ }^{13}$ BOE del 28 de abril de 1992. 
año se publica el Real Decreto 303/2010, de 15 de marzo, que vuelve a fijar, al amparo de la LOE, los requisitos mínimos de los centros pero con menos ambición y concreción, un hecho sorprendente por cuanto sería lógico que se tuviese en cuenta la necesidad de integrar las EEAASS en el EEES. A todo ello hay que sumar el que en ningún caso, a la hora de establecer la plantilla de los centros superiores, se hayan considerado perfiles profesionales propios en todas las actividades de apoyo a la docencia, la creación o la investigación, desde personal de bibliotecas a personal técnico de teatros y auditorios.

El único parámetro en el que los centros superiores de enseñanzas artísticas confluyen plenamente con el espíritu de Bolonia es en la relación numérica profesor/alumno, en tanto las ratios reducidas entre alumnado/profesorado ${ }^{14}$ potencian ese nuevo modelo de entender la docencia y el aprendizaje, bien lejos de la masificación en que ha derivado en la universidad española. Un aspecto positivo que convendría mantener y proteger. Por lo demás, es fácil deducir de todo lo anterior que la incorporación de las enseñanzas artísticas superiores al EEES ni estuvo acompañada de un plan específico de actuación ni se complementó con un necesario plan de financiación para atender las múltiples demandas derivadas de una convergencia que, en este caso, no va más allá de la equivalencia de títulos. Ni el Ministerio de Educación ni las Consejerías de Educación han elaborado una hoja de ruta para propiciar la integración de las EEAASS en el EEES, aunque los documentos generados en la ordenación de las enseñanzas señalen de forma precisa las responsabilidades de las administraciones educativas.

\section{Investigación y creación}

Todo ello tiene unas consecuencias nefastas para el desarrollo de la investigación en tanto los centros, que no están reconocidos como instituciones competentes en ese campo, no pueden desarrollar programas para demostrar su competitividad o captar fondos, ni ofrecer a sus titulados y tituladas la posibilidad de gozar de las becas y bolsas a que puede acceder el alumnado universitario. En esa dirección, no deja de ser un indicador importante de las oportunidades que se pierden, la transformación substantiva que han vivido los Institutos Superiores de Educación Física en su conversión en facultades universitarias, así como el número de revistas científicas propias de su área de conocimiento que todo ello ha generado. El portal DICE incluye un total de 37 revistas vinculadas al campo, de las cuales dos obtienen de la ANEP una calificación A+, y diez se consideran de tipo A.

La norma establece que los centros de EEAASS habrán de desarrollar programas de investigación en las disciplinas propias y al tiempo transitar hacia su integración en el Sistema Español de Ciencia y Tecnología, pero también abre la posibilidad de que

\footnotetext{
${ }^{14}$ Real Decreto 303/2010, de 15 de marzo, por el que se establecen los requisitos mínimos de los centros que impartan enseñanzas artísticas reguladas en la ley Orgánica 2/2006, de 3 de mayo, de Educación, publicado en el $B O E \mathrm{n}^{\circ} 86$, de 9 de abril.
} 
mediante convenios con las universidades puedan organizar estudios de doctorado específicos a las enseñanzas artísticas. En efecto, el RD 1614/2009, en su artículo 10, establece que las administraciones educativas "fomentarán convenios con las universidades para la organización de estudios de doctorado propios de las enseñanzas artísticas” (p. 89747), sin que se hayan arbitrado medidas específicas en esa dirección. Tampoco existe una normativa que permita que aquellos centros con un número suficiente de profesorado con título de doctorado, puedan conformar equipos de investigación al amparo de las convocatorias oficiales. Igualmente, el profesorado encuentra enormes dificultades para desarrollar o continuar su trayectoria creativa, y los centros carecen de la normativa y de los recursos necesarios para que ellos mismos puedan desarrollar líneas de trabajo vinculadas con el trinomio investigación, innovación y creación $(\mathrm{I}+\mathrm{i}+\mathrm{c})$, tan específico de estas enseñanzas.

\section{La garantía de calidad}

El RD 1614/2009 establecía la puesta en marcha de sistemas y procedimientos de evaluación periódica de la calidad de las EEAASS, en el contexto del EEES, con la finalidad de mejorar la actividad docente, investigadora y de gestión de los centros ( $p$. 89751). Todo ello implica la verificación periódica de las titulaciones y de los centros que las imparten, y que, en buena lógica, debieran contar con un Sistema de Garantía Interna de la Calidad, como ya establecen algunas Comunidades Autónomas ${ }^{15}$. El problema consiste en decidir hasta qué punto centros que son equiparables, en su organización, a institutos de secundaria podrán establecer y cumplir los protocolos propios de programas de acreditación y verificación como VERIFICA o DOCENTIA, y cuáles las herramientas y recursos para hacerlo así. Pero el problema también reside en el hecho de que hablemos de centros con muy escasa cultura y tradición en la evaluación del ejercicio docente del profesorado, en ocasiones sentida como un atentado contra una mal entendida "libertad de cátedra".

\section{Resultados y propuestas}

Marzal, en su análisis de la "anulación" de algunas disposiciones del RD 1614/2009, señalaba que la ordenación de las EEAASS es una de las "categorías más confusas de cuantas comprende el sistema educativo" derivado de la LOE, lo que genera "dificultades" a las EEAASS en su "relación con las enseñanzas universitarias" (2012, p. 44). Por ello se hace tan necesaria una ordenación que permita que el espíritu y la letra de la norma se cumplan, y que la relación entre la norma y su aplicación no devenga en aporía.

La propuesta del Ministerio de no abordar la vía universitaria ya contemplada en 1970, no ha ido acompañada de las normativas que garanticen esa plena convergencia

\footnotetext{
15 Así, en la Orden del 30 de septiembre de 2010 por la que se establece el plan de estudios de las enseñanzas artísticas superiores de grado en arte dramático en la Comunidad Autónoma de Galicia, publicado en el $D O G$, el 11 de octubre, p. 17087.
} 
de las EEAASS en el EEES y el cumplimiento y la superación de todas las funciones, responsabilidades y retos antes formulados. El hecho de que las administraciones educativas no hayan desarrollado una normativa que acompañe ese proceso de integración de las artísticas superiores en el EEES ${ }^{16}$, más allá de la creación de Institutos Superiores de Enseñanzas Artísticas que funcionan como unidades de gestión de centros, impide que éstos puedan desarrollar las nuevas funciones que ese marco implica. A día de hoy los centros superiores de enseñanzas artísticas, aún ofreciendo titulaciones equivalentes a grados universitarios en estudios catalogados como de "régimen especial", siguen funcionando como centros de "régimen general", lo que implica una integración en el EEES puramente nominal, con los prejuicios que genera al alumnado, al profesorado, a las enseñanzas y a la propia sociedad.

¿Qué hacer entonces? Pliego de Andrés (2014) analizaba algunas de las posibilidades que en estos últimos años se han venido formulando, que en buena medida coinciden con las presentadas por Pastor García (2012), y que Marzal (2010) analizaba para el caso de la música, pero considerando la ordenación de tales enseñanzas en Europa, en algunos casos matizando propuestas anteriores como las del profesor Embid Irujo (1997, 2002). En toda Europa las enseñanzas artísticas superiores o son universitarias o están adscritas a la universidad, con lo que en el horizonte asoma una posibilidad que la propia LOMCE contempla y que no es otra que la adscripción de los centros y de sus titulaciones a las universidades, camino de su integración definitiva. La posibilidad de la adscripción ya aparece recogida en la $\operatorname{LOU}^{19}$ en su artículo 11, que además establece que los centros adscritos se regirán "por lo dispuesto en esta Ley, por las normas dictadas por el Estado y las Comunidades Autónomas en el ejercicio de sus competencias, por el convenio de adscripción y por sus propias normas de organización y funcionamiento" (p. 49406). Se solventaría así de forma inmediata el problema de la normativa a desarrollar con carácter estatal y/o autonómico.

Quisiéramos concluir nuestra propuesta en defensa de la adscripción e integración universitaria de las EEAASS, resaltando la importancia que las mismas tienen en la formación de los trabajadores y trabajadoras del multiforme y muy extenso campo de la creación y la difusión cultural o de la conservación de nuestro patrimonio; destacamos también su transcendencia en la formación de profesionales y de investigadores que contribuyan a desarrollar plenamente todas las potencialidades y posibilidades del campo de la cultura como ámbito necesario en una economía sostenible, capaz de generar riqueza, diversificar el marcado laboral y contribuir al bienestar de la ciudadanía. Tal vez por eso en la inmensa mayoría de los países de nuestro entorno cultural las enseñanzas artísticas superiores forman parte de la oferta universitaria. Tomando como referencia universidades del prestigio de Brown, Columbia, Yale, Duke, Cornell, Dartmouth, Stanford o Carnegie Mellon, comprobamos que éstas, en su oferta educativa, incluyen grados propios en enseñanzas

\footnotetext{
${ }^{16}$ En la Comunidad Autónoma de Canarias sigue vigente la "Resolución de 25 de julio de 2005, por la que se dictan instrucciones generales sobre la organización y funcionamiento de los Centros Superiores de Enseñanzas Artísticas", ajena a la nueva ordenación de las enseñanzas y a su integración en el EEES. BOC $\mathrm{n}^{\circ} 162$, de 19 de agosto.

${ }^{17}$ BOE del 24 de diciembre de 2001.
} 
artísticas, además de estudios de posgrado. Un ejemplo a seguir en esa convergencia que supone el EEES, pues como demuestra la experiencia de tales instituciones, la integración universitaria es la mejor opción para alcanzar los objetivos demandados por la legislación vigente. Una opción nada costosa, viable y, sobre todo, rápida, como se ha mostrado en casos que, en apariencia, pudieran parecer más complicados, como el de la enseñanza militar (Romero Serrano, 2014). Una opción que permitiría que las EEAASS pudiesen convivir bajo un mismo techo trenzando redes, promoviendo sinergias y desarrollando proyectos. Como en Europa, como en el mundo.

\section{Referencias bibliográficas}

ACESEA (2010). Las enseñanzas artísticas superiores. Legislación. Murcia: Nausícaä Edición electrónica. Recuperado de: http://www.acesea.es/www/files/Libro-2010DEF.pdf

ACESEA (2012). Las enseñanzas artísticas en el espacio europeo de educación superior. Situación y perspectivas. Recuperado de: http://www.profesionalesdanza.com/en/documentos/documento_de_trabajo_jornada s_acesea

ÁLVAREZ GONZÁLEZ, M. (2008). La tutoría académica en el Espacio Europeo de Educación Superior. Revista Interuniversitaria de Formación del Profesorado, 61, 71-88.

ÁlVAREZ PÉREZ, P. R. y GONZÁlEZ AFONSO, M. C. (2008). Análisis y valoración conceptual sobre las modalidades de tutoría universitaria en el Espacio Europeo de Educación Superior. Revista Interuniversitaria de Formación del Profesorado, 61, 49-70.

ARENAS, M. G., HERRERA, L. J. y DÍAZ, J. (2012). Cálculo y valor del crédito ECTS para el profesorado. Enseñanza y Aprendizaje de la Ingeniería de Computadores, 2, 51-71.

BAS PALMERO, G. (2014). Evolución histórica de los estudios superiores de danza en España. El caso de la Comunidad Valenciana. Danzaratte, 8, 5-19.

CALVET VILLENA, G. (2013). Titulacions artístiques superiors. Víctimes dels buits legislatius. Entreacte, 182, 8-10.

CARRERA, F. (2014). Una plataforma muy estable. Danzaratte, 8, 77-93.

CASTRO, R. (1969). Incorporación de los conservatorios o escuelas superiores de música a la universidad. Cuadernos de actualidad artística, 6, 42-52.

CSEA (2011): Informe anual sobre el estado y situación de las Enseñanzas Artísticas. Curso 2009-2010. Recuperado de: https://sede.educacion.gob.es/publiventa/detalle.action?cod=14678

EMBID IRUJO, A. (1997). Informe sobre la conveniencia de promulgar una Ley Orgánica Reguladora de la Organización en Régimen de Autonomía de las 
Enseñanzas Artísticas Superiores en España. Madrid: ACESEA. Recuperado de: http://www.acesea.es/www/files/informe1997.pdf

EMBID IRUJO, A. (2002). Problemática actual de las enseñanzas artísticas superiores en España. En Serrano Masegoso, J. A. (Ed.) Los Centros Superiores de Enseñanzas Artísticas y la reforma de las Enseñanzas en España, 23-61. Murcia: ACESEA.

FONT, J. (2010). Crónica de un trayecto. En ACESEA (Ed.), Las enseñanzas artísticas superiores. Legislación, 12-17. Murcia: Nausícaä Edición electrónica. Recuperado de: http://www.acesea.es/www/files/Libro-2010-DEF.pdf

GAIRÍN, J., FEIXAS, M., GUILLAMÓN, C. y QUINQUER, D. (2004). La tutoría académica en el escenario europeo de la Educación Superior. Revista Interuniversitaria de Formación del Profesorado, 49, 61-78.

ISEACV (2013). Instrucciones de 9 de septiembre de 2013 del Instituto Superior de Enseñanzas Artísticas de la Comunitat Valenciana para el curso 2013-2014. Recuperado de: http://www.iseacv.es/web_cst/isea1_4.php

LEMES CASTELLANO, F. L. (2012). Antecedentes sobre la ordenación de las enseñanzas artísticas superiores en España. En ACESEA (Ed.) Las enseñanzas artísticas en el espacio europeo de educación superior. Situación y perspectivas, 720.

Recuperado

de: http://www.profesionalesdanza.com/en/documentos/documento_de_trabajo_jornada s_acesea

LÓPEZ, J. (2010). La LOE. Un salto cualitativo para las enseñanzas artísticas. En LÓPEZ, J. y VICO, M. L. (Coords.) Las Enseñanzas Artísticas Superiores en el Espacio Europeo de Educación Superior, 9-20. Madrid: Ministerio de Educación.

LÓPEZ, J. y VICO, M. L. (Coords.) (2010). Las Enseñanzas Artísticas Superiores en el Espacio Europeo de Educación Superior. Madrid: Ministerio de Educación.

LUNA, C. (2014). La adscripción de los centros de enseñanzas artísticas superiores en la Universidad. Danzaratte, 8, 94-103.

MARZAL, R. (2010). El régimen jurídico de las enseñanzas musicales. Valencia: Diputación de Valencia.

MARZAL, R. (2012). Las enseñanzas artísticas superiores en el sistema educativo general. Justicia administrativa, 57, 43-74.

MECD (2003). La integración del sistema universitario español en el Espacio Europeo de Educación Superior. Madrid: Ministerio de Educación, Cultura y Deporte. Disponible en: http://www.eees.es/pdf/Documento-Marco_10_Febrero.pdf

MENÉNDEZ VARELA, J. L. (2009). La aplicación del Sistema Europeo de Transferencia y Acumulación de Créditos. Revista Complutense de Educación, 20 (2), 381-401. 
MOLARES, O. A., RINCÓN, A. G. y ROMERO, J. T. (2005). Cómo enseñar a investigar en la universidad. Educere, 29, 217-224.

PASTOR GARCÍA, V. (2012). Informe sobre los posibles modelos de gestión y organización de las EEAASS. En ACESEA (Ed.) Las enseñanzas artísticas en el espacio europeo de educación superior. Situación y perspectivas, 59-67. Recuperado de: http://www.profesionalesdanza.com/en/documentos/documento_de_trabajo_jornada S_acesea

PASTOR GORDERO, P. (2012). La música a la universidad: por la integración en la universidad de las enseñanzas artísticas superiores. Eufonía. Didáctica de la música, 56, 52-70.

PÉREZ GARCÍA, M. M. y SICILIA CAMACHO, A. (2011). El proceso de convergencia de las enseñanzas de arte dramático y danza en el Espacio Europeo de Educación Superior. Psychology, Society \& Education, 3 (2), 133-145.

PLIEGO DE ANDRÉS, V. (2014). Oportunidad y conveniencia de integrar las enseñanzas artísticas superiores en la universidad. Danzaratte, 8, 104-119.

ROMERO SERRANO, J. (2014). El Proceso de Bolonia y la integración de la enseñanza militar en el sistema educativo general. Un nuevo paso. Arbor, 765. Recuperado de: http://arbor.revistas.csic.es/index.php/arbor/article/view/1902/2123

RUÉ DOMINGO, J. (2004). La convergencia europea: entre decir e intentar hacer. Revista Interuniversitaria de Formación del Profesorado, 49, 39-60.

VIEITES, M. F. (2015a). La investigación teatral en una perspectiva educativa: retos y posibilidades. Educatio Siglo XXI, 33 (2), 11-30.

VIEITES, M. F. (2015b). Arte dramático y universidad pública. Hacia una integración necesaria. Profesorado, Revista de Currículum y formación del profesorado, 19 (1), 555-573

ZABALZA, M. A. (2008a). Innovación en la Enseñanza Universitaria: el proceso de convergencia hacia un Espacio Europeo de Educación Superior. Educação, 31 (3), 199-209.

ZABALZA, M. A. (2008b). El Espacio Europeo de Educación Superior: innovación en la enseñanza universitaria. Innovación Educativa, 18, 69-95.

ZALDÍVAR, A. (2005). Las enseñanzas musicales y el nuevo Espacio Europeo de Educación Superior: el reto de un marco organizativo adecuado y la necesidad de la investigación creativa y performativa. Revista Interuniversitaria de Formación del Profesorado, 52, 95-122. 


\section{Correspondencia con el autor}

Manuel Francisco VIEITES

Escuela Superior de Arte Dramático de Galicia

Poza Cabalo s/n

36209 Vigo, Pontevedra

e-mail: mvieites@uvigo.es 